\title{
Impact of the Coronavirus Disease Pandemic on Health-Related Quality of Life of Patients with Inflammatory Bowel Disease
}

\author{
Emma Paulides ${ }^{1}$ (1) $\cdot$ Annelieke Pasma ${ }^{2} \cdot$ Nicole S. Erler $^{3} \cdot$ Rachel L. A. van Eijk $^{1} \cdot$ Annemarie C. de Vries $^{1}$. \\ C. Janneke van der Woude ${ }^{1}$
}

Received: 5 March 2021 / Accepted: 14 June 2021 / Published online: 28 June 2021

(c) The Author(s) 2021

\begin{abstract}
Background To learn from the crisis caused by the coronavirus disease (COVID-19) pandemic and be prepared for future pandemics, it is important to investigate the impact of this period on the wellbeing of patients with inflammatory bowel disease (IBD).

Aims To describe the health-related quality of life (HRQoL) and disease control of IBD patients during the first wave of the COVID-19 pandemic in The Netherlands.

Methods Between March 17 and July 1, 2020, patients aged 18 years and older with IBD from the Erasmus MC (Rotterdam, The Netherlands) were invited to complete online questionnaires at week 0, 2, 6 and 12. The Inflammatory Bowel Disease Questionnaire (IBDQ), the Inflammatory Bowel Disease Control-8 (IBD-control-8) and the numeric rating scale on fatigue were used. The evolution of the different outcomes over time was measured using mixed models.

Results Of 1151 invited patients, 851 patients (67\% CD and 33\% UC or IBD-U) participated in the study (response rate 74\%). No relevant changes in total scores were found over time for the IBDQ (effect estimate 0.006, 95\% CI [- 0.003 to 0.015 ]) and IBD-control-8 (effect estimate 0.004, 95\% CI [0.998-1.011]). There was a slight, increasing trend in fatigue scores over time (effect estimate $0.011,95 \%$ CI [0.004, 0.019]).

Conclusions This first lock down due to the COVID-19 pandemic in The Netherlands did not impact on the HRQoL and disease control of patients with IBD. Up to date information may have contributed to a stable HRQoL in IBD patients even in an extreme period with restrictions and insecurities.
\end{abstract}

Keywords Inflammatory bowel disease · COVID-19 · Pandemic · Quality of life · Disease control · Fatigue

Disclosures: Professor C.J. van der Woude has served on advisory boards for Abbvie, Takeda, Pfizer and Celltrion. She is supported by research funding from ZonMW, Tramedico, and Pfizer. Dr A.C. de Vries has participated in advisory board and/or received financial compensation from the following companies: Jansen, Takeda, Abbvie and Tramedico.

Emma Paulides

e.paulides@erasmusmc.nl

1 Department of Gastroenterology and Hepatology, Erasmus University Medical Center, Doctor Molewaterplein 40, 3015 GD Rotterdam, The Netherlands

2 Department of Rheumatology, Erasmus University Medical Centre, Rotterdam, The Netherlands

3 Department of Biostatistics, Erasmus University Medical Centre, Rotterdam, The Netherlands

\section{Introduction}

In December 2019, the first patients were diagnosed with pneumonia caused by a novel coronavirus (SARS-Cov-2) in Wuhan, China. This pathogen has phylogenetic similarities to SARS-CoV and causes Corona Virus Disease 2019 (COVID-19) [1, 2]. Signs of infection include fever, cough and dyspnea, but in more severe cases the infection results in pneumonia, severe acute respiratory syndrome and even death [3]. The pathological features of lung tissue of deceased patients that were tested SARS-Cov-2 positive show diffuse alveolar damage, inflammation and extensive lung thrombosis, providing a target for therapy $[4,5]$. In The Netherlands, the first confirmed case of COVID-19 was reported on February 27, 2020. On March 12, 2020, national quarantine measures were taken and these were expanded 
on March 23, resulting in a targeted lockdown, known as "intelligent" lockdown, of the country [6, 7].

At the time of the outbreak in The Netherlands, it was unknown yet how this virus would impact patients that are suffering from a chronic condition. Inflammatory Bowel Disease (IBD) consists of Crohn's disease (CD) and ulcerative colitis (UC) and is associated with different aspects that contribute to an increased risk of opportunistic infections, such as an altered immune response, malnutrition, surgery and immunosuppressive medication [8-11]. Moreover, there were concerns that IBD patients were at increased risk for SARS-CoV-2-induced infection because coronaviruses bind to angiotensin-converting enzyme 2 , which is present in high concentrations in the terminal ileum and colon [12].

As patients with IBD already have a lowered healthrelated quality of life (HRQoL) compared to the general population, we hypothesized that this uncertain period would impact on their physical and mental wellbeing [13, 14]. Fear and worries about an increased susceptibility to infection, higher risk for worse outcome and the measures taken may thereby have had a negative effect on the lives of patients with IBD. In addition, psychosocial dysfunction and stress can have a negative effect on bowel symptoms and disease activity through the bidirectional communication between the gut and the brain [15] To make a period with wide-ranging measures as tolerable as possible, communication is of great importance by explaining people what is happening and why, and how long it is expected to last [16].

Therefore, the aim of this research was to describe the self-reported HRQoL and disease control of patients with IBD from the start of the COVID-19 pandemic during different phases of restrictions in The Netherlands.

\section{Materials and Methods}

\section{Objective}

The objective of our study was to describe the HRQoL and disease control of patients with IBD during the first wave of the COVID-19 pandemic and measures in The Netherlands, while keeping patients informed about new insights.

\section{Study Design}

On March 17, all patients from the Erasmus Medical Center in Rotterdam, the Netherlands, aged 18 years and older that had a diagnose treatment code of IBD, CD or UC or IBD unclassified (IBD-U) and were seen in the outpatient clinic from March 2019 to February 2020 with a known email address were informed about COVID-19 by the IBD team. A newsletter was sent to the patients via email that contained general health instructions, the announcement that outpatients' visits were replaced by telephone or video consults if possible and the postponement of elective endoscopic procedures. The letter also included specific instructions on maintenance of drugs, information on the continuation of drug infusions at the hospital, and patients using prednisone were asked to contact the IBD team to discuss how to taper off. A dedicated email address was made for communication regarding the coronavirus. Patients were asked to fill out a diary to register airway complaints as part of standard of care.

After one week, patients were invited to participate in the study by filling out questionnaires at multiple time points. A link was sent via email at week $0,2,6$ and 12 to complete the survey. If patients did not want to participate, they still received the symptom diary and were given the same care as usual. Patients were excluded if they did not fill out any of the questionnaires. After two weeks, patients were invited to a webinar where a specialist team of local IBD doctors answered questions from patients about COVID-19. See Fig. 1 for an overview of the taken measures and study procedures in time.

\section{Instruments}

The Inflammatory Bowel Disease Questionnaire (IBDQ) is an IBD specific tool to measure HRQoL that exists of 32 questions divided into four domains: bowel and systemic symptoms, social and emotional functioning. Each question

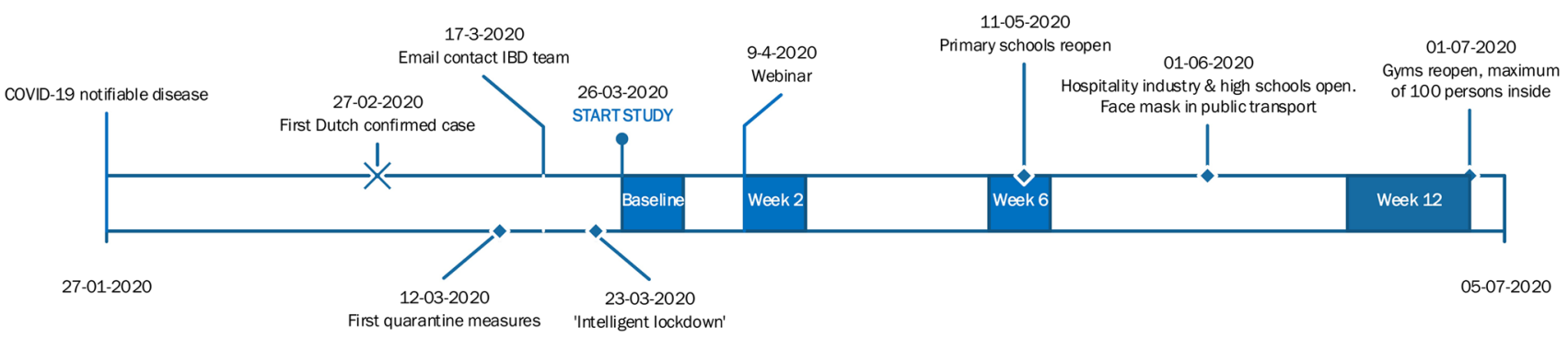

Fig. 1 Timeline of measures in The Netherlands 
can be answered on a 7-point rating scale. Scores range from 32 to 224 where higher scores indicate better HRQoL [17, $18]$.

The Inflammatory Bowel Disease control-8 (IBD-control-8) is a self-administered 8-item questionnaire with three answers options, measuring disease control over the past two weeks. Scores range from 0 to 16 , with higher scores indicating better disease control [19].

The Numeric Rating Scale (NRS) for fatigue is a singleitem measure assessing gravity of fatigue over the past week. The scale ranges from 0 to 10; 0 indicates no fatigue at all and 10 the worst fatigue [20].

\section{Statistics}

Since the recent outbreak of COVID-19 and lack of epidemiological data in the Netherlands, we aimed to include all patients with IBD from the Erasmus MC. Therefore, no sample size calculation was made. Data are presented as count and percentage for categorical variables, mean and standard deviation for continuous, approximately normally distributed variables, and median and interquartile range for non-normal continuous data.

For the assessment of the influence of time on the different outcomes, scores were investigated by analysis of repeated measurements. Mixed-effects models were fitted that included time (in weeks since baseline) as fixed effect, and a random intercept to take into account the correlation between repeated measurements within the same patient.

The evolution of the IBDQ total score over time was measured using a linear mixed model. To better comply with the assumption of approximately normally distributed residuals, the outcome was transformed using the square root of the inverted score $(\sqrt{225-\text { IBDQ total score }})$. For this outcome, we fitted an additional model that also included the patients' sex, diagnosis (CD vs. UC), age, whether they had a stoma (no vs yes), interactions between these variables and time, and medication use (no, biological or other). To facilitate interpretation of the results, the expected scores at baseline, 2, 6 and 12 weeks were transformed back to the original scale and plotted together with the original data. The expected values of IBDQ and corresponding 95\% confidence intervals are indicated by the vertical line and shaded bar around it. These confidence intervals were obtained using bootstrap.

Changes in the IBD-control-8 total score over time were analyzed using a Gamma mixed model with a log-link, in which the outcome scale was reversed. Results are presented on the scale of the transformed outcome and visualized by plotting the expected values per time point transformed back to the original scale of the data.

The evolution of the fatigue score over time was explored using a beta mixed model with a logit link. The fatigue score was transformed to have values between zero and one by subtracting 0.5 and dividing by 10 . Results are presented as odds ratios and corresponding $95 \%$ confidence intervals and visualized on the original scale of the data.

$P$ values $<0.05$ were considered statistically significant. All calculations were performed in $\mathrm{R}$ version 4.0.3 (2020-10-10) [21] and using the packages lme4 (1.1.26) [22], GLMMadaptive (0.7.15) [23] and glmmTMB (1.0.2.1) [24].

\section{Results}

\section{Study Cohort}

One thousand one hundred fifty-one patients were invited in the study of whom 851 patients participated (response rate $74 \%$ ). Three hundred patients did not take part for reasons specified in Fig. 2. Of 851 patients with IBD (median age 41 years; female $57 \%$ ), $67 \%$ had CD and $33 \%$ UC or IBD-U. Patient characteristics are summarized in Table 1.

\section{Outcomes}

Overall, there was no evidence for relevant changes of the mean QoL over time as measured by the IBDQ (effect estimate $0.006,95 \%$ CI [ -0.003 to 0.015$]$ ), see Supplementary Table 1 and Fig. 3. Taking into account patient characteristics, however, showed that female sex was associated with somewhat lower IBDQ scores at baseline as compared to males (i.e., larger values on the transformed scale; effect estimate: $0.518,95 \%$ CI $[0.201,0.834]$ ) and that IBDQ scores in females declined over time (effect on the transformed scale: $0.020,95 \%$ CI [0.001, 0.039]), while they remained relatively constant in males (Fig. 4a). A diagnosis of UC was associated with higher IBDQ scores (i.e., smaller values on the transformed scale; effect estimate: $-0.509,95 \% \mathrm{CI}$ $[-0.852,-0.166])$ without a change over time (Fig. 4b). There was no evidence for an effect of age, medication use or presence of stoma on the IBDQ score at baseline, nor that there were relevant changes of these effects over time (see Supplementary Table 2).

In addition, there was no evidence for relevant changes of disease control over time as measured by the IBD-control- 8 (effect estimate 0.004, 95\% CI [0.998-1.011]), see Supplementary Table 3 . The expected values of the IBD-control-8 and corresponding 95\% confidence intervals are indicated by the vertical line and shaded bar around it (Fig. 5).

There was a slight, increasing trend in fatigue scores over time (effect estimate from the beta mixed model $0.011,95 \%$ CI [0.004, 0.019]; Supplementary Table 4). This effect translates to an estimated fatigue score at baseline of 5.956 (95\% 


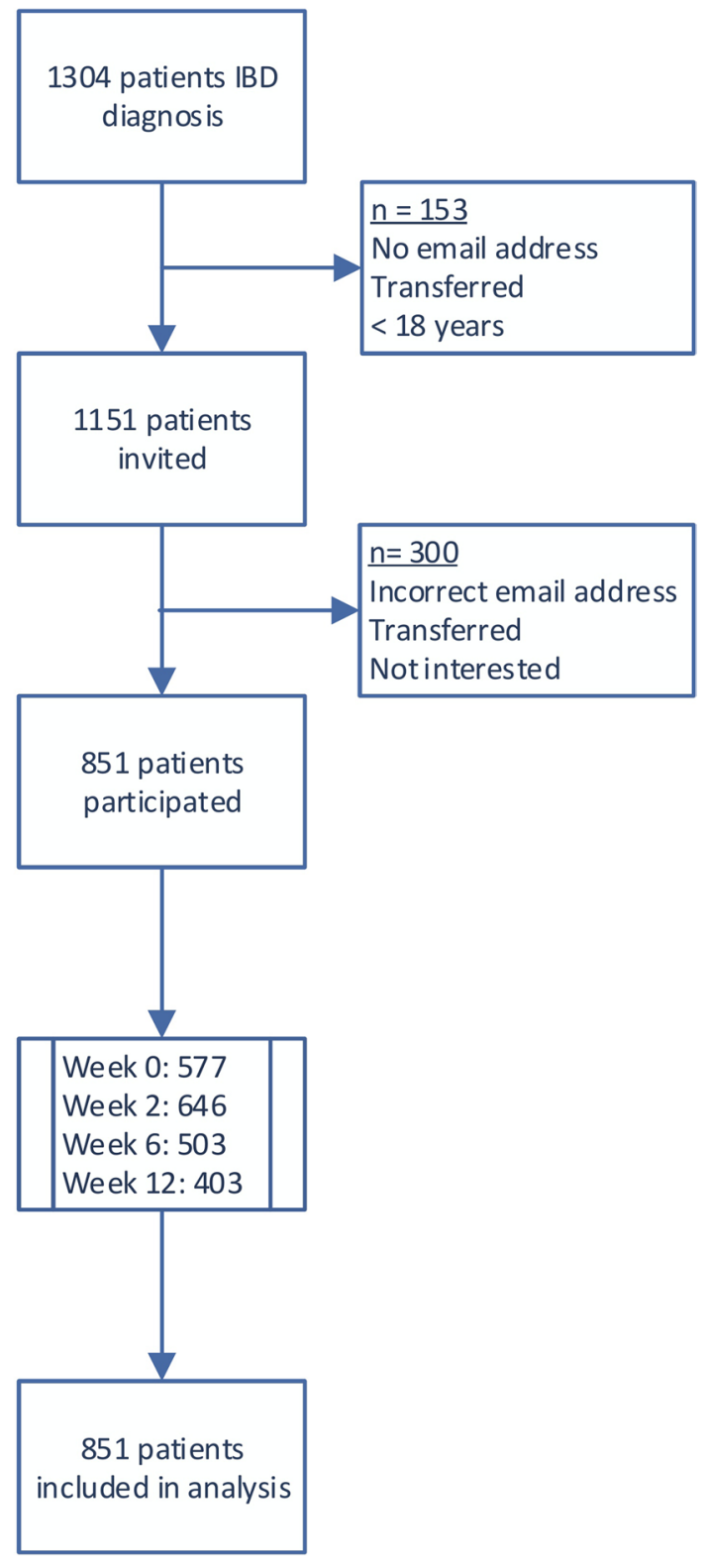

Fig. 2 Flowchart of patients through study

CI $[5.751,6.159])$ that increases to 6.285 (95\% CI [6.044, 6.523]) in week 12 (Fig. 6).

\section{Discussion}

This is the first large cohort study investigating HRQoL and self-reported disease control of patients with IBD during the first wave of the COVID-19 pandemic over time. Results show a relatively high HRQoL and disease control of the IBD patient population, which is in line with the results of two large population-based cohorts in Switzerland and Canada $[25,26]$. Although previous studies have reported that
Table 1 Baseline characteristics of 851 patients with IBD

\begin{tabular}{|c|c|}
\hline Characteristics & $n(\%)$ \\
\hline \multicolumn{2}{|l|}{ Sex } \\
\hline Female & $488(57)$ \\
\hline Male & $363(43)$ \\
\hline Age (year), median & 41 \\
\hline \multicolumn{2}{|l|}{ Type of IBD } \\
\hline $\mathrm{CD}$ & $567(67)$ \\
\hline $\mathrm{UC}$ or IBD-U & $284(33)$ \\
\hline \multicolumn{2}{|l|}{ Current IBD medication } \\
\hline None & $199(23)$ \\
\hline Aminosalicylates & $171(20)$ \\
\hline Steroids & $123(15)$ \\
\hline Thiopurines & $121(15)$ \\
\hline Methotrexate & $13(1.5)$ \\
\hline Calcineurin inhibitors & $6(0.7)$ \\
\hline Biologicals & $365(43)$ \\
\hline JAK-Stat inhibitors & $10(1)$ \\
\hline Naltrexone & $1(0.1)$ \\
\hline Stoma & $104(12)$ \\
\hline \multicolumn{2}{|l|}{ Age at diagnosis CD } \\
\hline$<16$ years & $101(18)$ \\
\hline $17-40$ years & $408(72)$ \\
\hline$>40$ years & $58(10)$ \\
\hline \multicolumn{2}{|l|}{ CD location } \\
\hline Ileitis & $134(24)$ \\
\hline Colitis & $117(20)$ \\
\hline Ileocolitis & $316(56)$ \\
\hline Upper GI & $58(10)$ \\
\hline \multicolumn{2}{|l|}{ Disease behavior } \\
\hline Luminal disease & $298(53)$ \\
\hline Stricturing disease & $181(32)$ \\
\hline Penetrating disease & $121(21)$ \\
\hline Perianal involvement & $121(21)$ \\
\hline \multicolumn{2}{|l|}{ UC extent (+ IBD-U) } \\
\hline Proctitis & $20(7)$ \\
\hline Left sided colitis & $96(34)$ \\
\hline Pancolitis & $168(59)$ \\
\hline
\end{tabular}

$n$ number, $C D$ Crohn's disease, $U C$ ulcerative colitis, $I B D-U$ inflammatory bowel disease unclassified, GI gastrointestinal, JAK-STAT inhibitor Janus kinase signal transducer and activator of transcription proteins inhibitor

IBD patients were more afraid of being infected with SARSCoV-2 than healthy controls and feared a negative effect of their medication, this did not lead to impaired HRQoL or disease-control in our population [27, 28]. HRQoL remained mostly stable during different phases of restrictions, and there was no difference between adolescents and older patients regarding HRQoL in general and changes over time. A possible explanation for this could be social distancing and staying at home, which for a significant part of our 
Fig. 3 The IBDQ total scores per time point with corresponding $95 \%$ confidence intervals

Fig. 4 The effect of sex, age and diagnosis on the IBDQ score over time, under the assumption that patients had no stoma and received biological therapy. a

The association between age (IQR) and sex and IBDQ score, and interactions between these variables over time. b The association between diagnosis (CD vs. UC) and sex and IBDQ score, and interactions between these variables over time

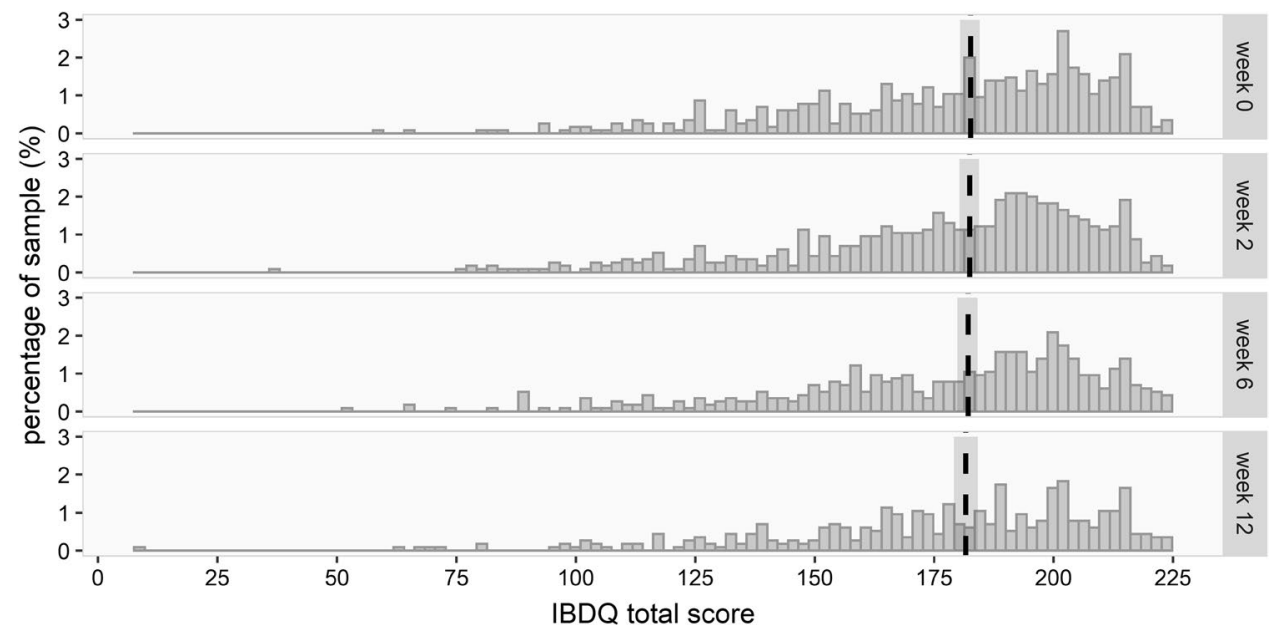

A age: $\rightarrow 30(\mathrm{Q} 1) \rightarrow 41$ (median) $\rightarrow 54(\mathrm{Q} 3)$

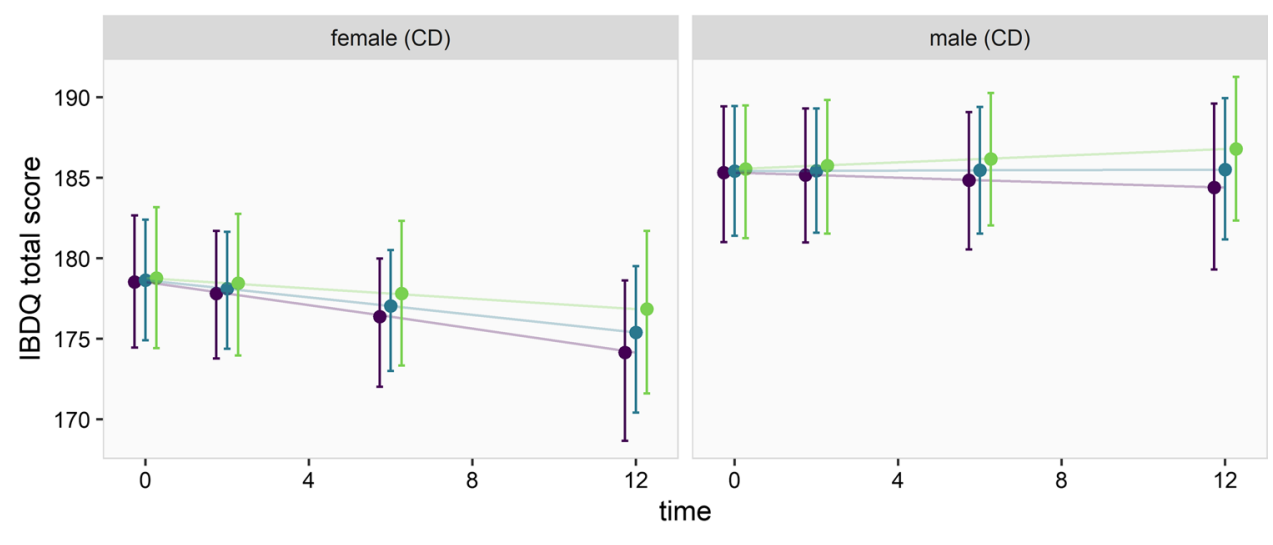

B diagnosis: $\triangle \mathrm{CD} \bullet \mathrm{UC}$

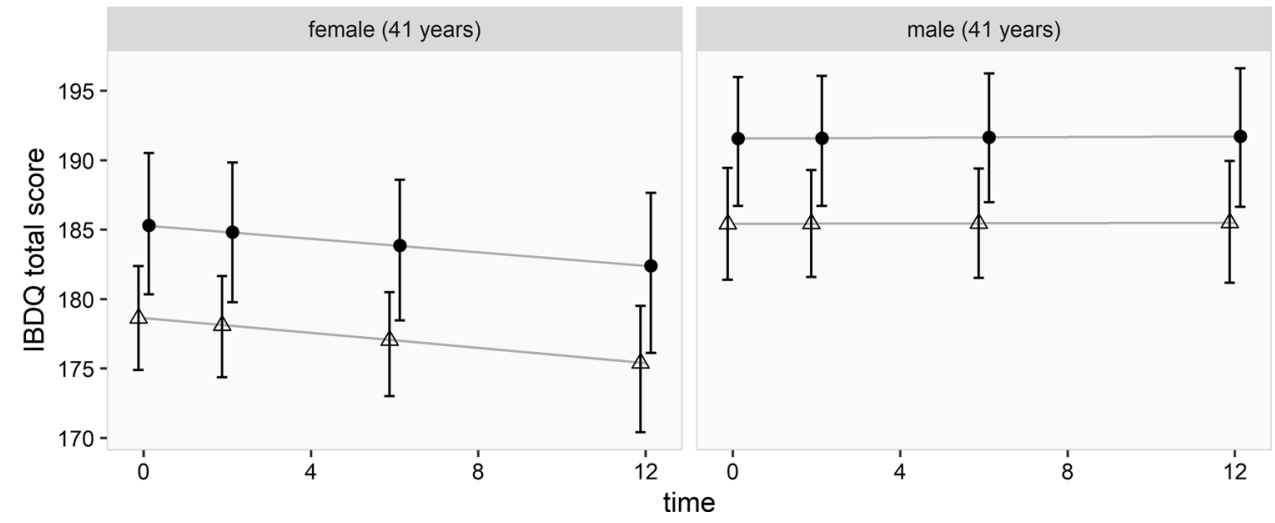

patient population, is already an everyday issue because of uncontrolled bowel movements, pain and fatigue [29]. However, for patients with a chronic disease, social support can be of great importance when struggling to try to stay safe while managing the disease.

At our department, we decided to inform and educate our patients at an early stage by sending newsletters, organizing a webinar and being reachable by email every day of the week. An anonymous web survey completed by 3815 patients with IBD worldwide collected data on the patients' views on the information flow during the early phase of the pandemic. Approximately half of the respondents reported receiving information about COVID-19 from their specialist (923/2116) or specific recommendations to prevent infection 
Fig. 5 The IBD-control-8 total scores per time point with corresponding $95 \%$ confidence intervals

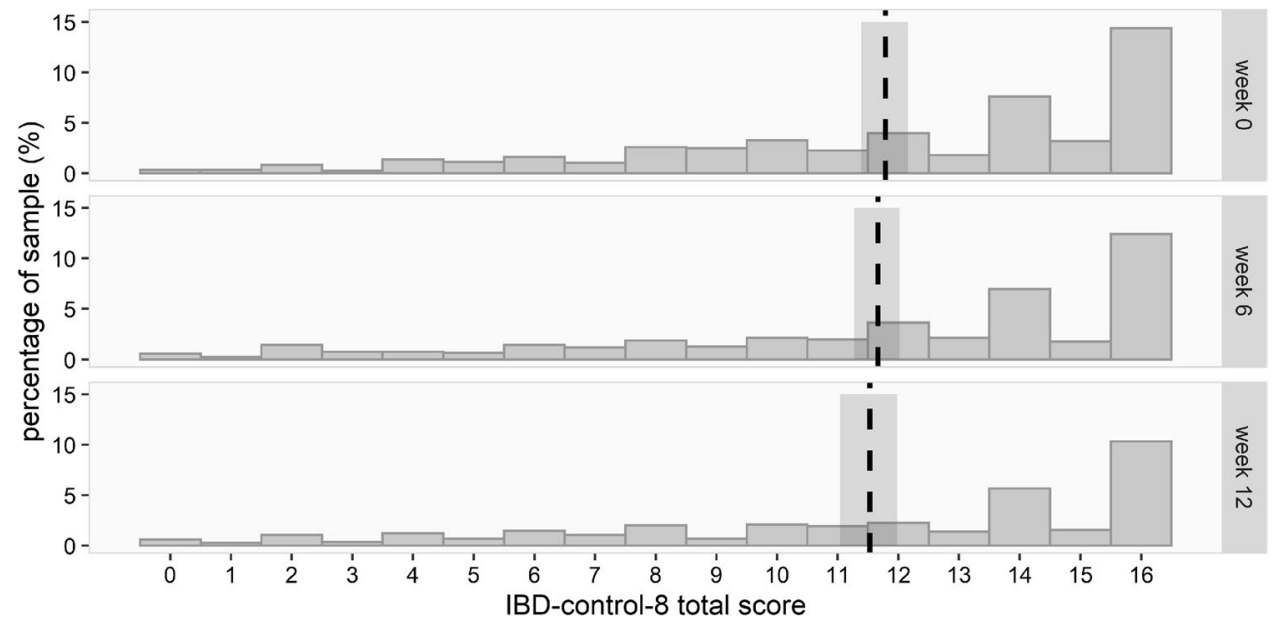

Fig. 6 The NRS fatigue scores per time point with $95 \%$ confidence intervals

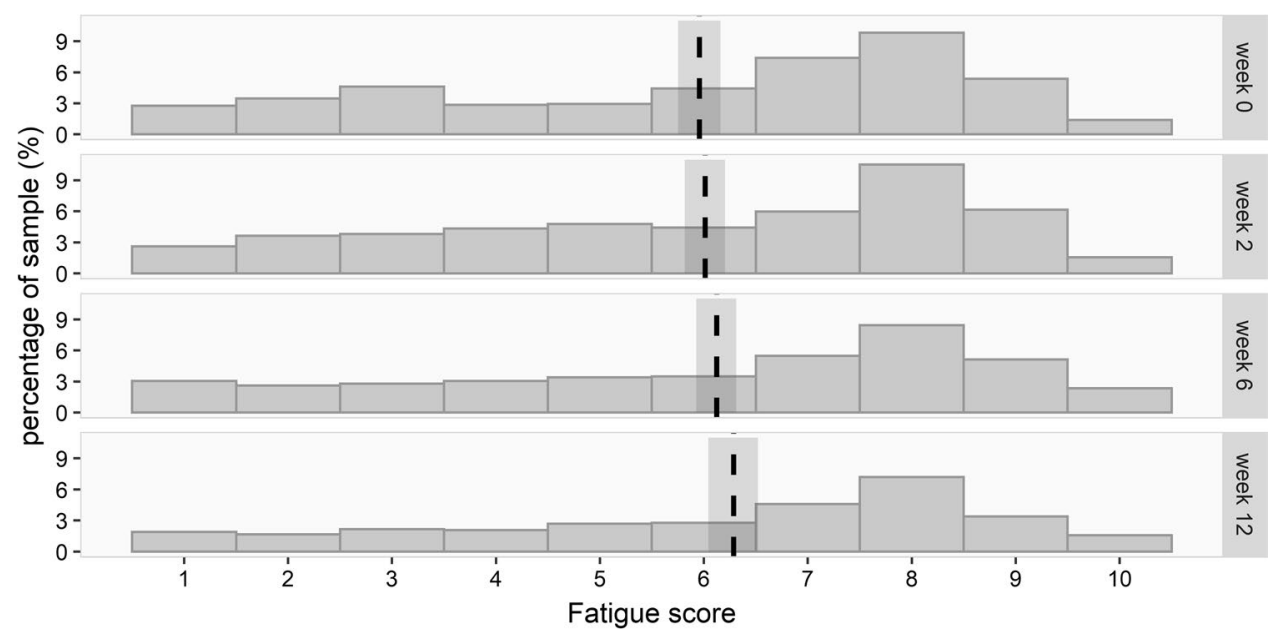

(1133/2172). Most patients would have preferred to receive more recommendations regarding COVID-19 from their physician (1184/1991), and about a quarter of them were not satisfied with the given advice (300/1304) [28]. This highlights the need for adequate information and could, in our cohort, have contributed to good and stable HRQoL.

Because of the pandemic, face-to-face outpatient visits were cancelled and replaced with telephone and video consultations. Furthermore, diagnostic procedures were delayed; priority was given to patients with symptoms or signs of relapse. Healthcare professionals and institutions were therefore forced to think of other ways to monitor patients outside the hospital. This study showed that, despite the change in care, overall HRQoL was good and remained mostly stable over time. Moreover, a cross-sectional study in 171 IBD patients in Madrid reported a high degree of satisfaction of outpatients with remote consultations, with no differences between scheduled and urgent consultations $(p<0.82)$. Less than $20 \%$ stated that they would have preferred a face-to-face visit to the telephone visit [30]. Therefore, this pandemic could be an opportunity to think about incorporating telehealth and remote patient monitoring in chronic disease management. In addition, telemedicine can lead to maintaining independence and minimizing travel costs and time missed at work [31].

Currently, two studies reported on HRQoL of IBD patients during COVID-19. In a small-sized study by Azzam et al., 29 IBD patients from a tertiary care center in Saudi Arabia were questioned in November 2019 and March 2020. There was no difference between the 10-item IBD-disk variables pre- and post- pandemic, and so it was concluded that the current COVID-19 pandemic did not impact on disability [32]. In Hubei Province, in China, 102 patients participated in a cross-sectional study, of which $85 \%$ reported a good QoL (measured by the SIBDQ) and $15 \%$ a poor QoL [33]. In another immune-mediated disease, the impact of COVID-19 on HRQoL was also assessed. One hundred-fifty-eight Italian patients affected by primary antibody deficiencies were switched to remote assistance with home treatment during the COVID-19 pandemic. HRQoL and psychological distress were surveyed and compared to data from 2017. QoL did not vary significantly from pre-pandemic $(p=0.804)$ and 
the remote assistance program and the shift from hospital- to home-based treatment did not impact on anxiety and depression ( $p=0.752)$ [34]. Overall, these conclusions are in line with the results of our study.

This study has some limitations. No data on disease activity and its impact on HRQoL over time were available in our cohort. Nevertheless, the IBD-control-8 is shown to have a moderate to strong correlation with the disease activity measures the Harvey-Bradshaw index (HBI) for Crohn's disease and the Simple Clinical Colitis Index (SCCAI) for ulcerative colitis [19]. Therefore, this questionnaire was sufficient to detect the worsening of the disease. Another limitation is that participants did not always fill out all questionnaires. The statistical models applied here are valid if missing values in the response are random, although this assumption needs to be considered carefully. A possible explanation for the missing data is the relatively short availability of the invitation link and the communication of the study via email. Furthermore, no data on quarantine behavior of individual patients were available. Work impairment, reduced social and physical contact with others and loss of routine could have played a major role in the impact of the pandemic on HRQoL. As a result, quarantine and avoidance behavior as a predictor for the psychological and physical impact of the pandemic could not be investigated.

It is foreseen that SARS-Cov-2 will be with us for a longer period of time, and that it can become a seasonal illness just like the influenza virus. Therefore, it is important to focus on mental health together with physical health to be able to define the impact of COVID-19 on patients with IBD and detect changes in their wellbeing over time. Infection rates, treatment options, organization of care and opportunities to use telehealth are different for countries around the world, highlighting the need for more research on HRQoL and COVID-19 in IBD. Information on patient characteristics such as co-morbidities, medication use, disease duration and mental health care use can contribute to identifying a vulnerable subgroup of patients.

\section{Conclusion}

This large cohort study showed that HRQoL and selfreported disease control in the outpatient population were good and remained stable during the COVID-19 pandemic despite the measures. Possible explanations for this positive result are the targeted lockdown, known as "intelligent" lockdown, the effect of the instructions given as well as the approachability of the IBD-team.

Supplementary Information The online version contains supplementary material available at https://doi.org/10.1007/s10620-021-07118-8.
Acknowledgments The authors wish to thank all members of the IBD team and the Biological Registry for monitoring the patients with IBD and helping with generating data.

Author's contribution Conception and design of the study were contributed by C.J.W., A.C.V., E.P., R.L.A.E., N.S.E. Acquisition of data was contributed by E.P., C.J.W., R.L.A.E., A.P., A.C.V. Analysis and interpretation of data were contributed by E.P., N.S.E., A.P., C.J.W. Drafting article and revising it critically were contributed by E.P., C.J.W., A.C.V., A.P., N.S.E., R.L.A.E. Final approval of the version was contributed by E.P., C.J.W., A.C.V., A.P., N.S.E., R.L.A.E.. All authors have made substantial contributions to the conception and design of the study, the acquisition of data, the analysis and interpretation of data or drafting the article or revising it critically for important intellectual content. All authors gave final approval of the version to be submitted.

\section{Declarations}

Conflict of interest There is no potential conflict of interest for this work for all authors.

Ethics approval All procedures were in accordance with the ethical standards of the institutional and/or national research committee and with the 1964 Declaration of Helsinki and its later amendments or comparable ethical standards. Ethical approval was received by the Ethical Committee of the Erasmus MC (MEC-2020-0310).

Consent to participate All subject gave their electronic informed consent.

Open Access This article is licensed under a Creative Commons Attribution-NonCommercial 4.0 International License, which permits any non-commercial use, sharing, adaptation, distribution and reproduction in any medium or format, as long as you give appropriate credit to the original author(s) and the source, provide a link to the Creative Commons licence, and indicate if changes were made. The images or other third party material in this article are included in the article's Creative Commons licence, unless indicated otherwise in a credit line to the material. If material is not included in the article's Creative Commons licence and your intended use is not permitted by statutory regulation or exceeds the permitted use, you will need to obtain permission directly from the copyright holder. To view a copy of this licence, visit http://creativecommons.org/licenses/by-nc/4.0/.

\section{References}

1. Guan WJ, Ni ZY, Hu Y, et al. Clinical characteristics of coronavirus disease 2019 in China. N Engl J Med 2020;382:1708-1720.

2. Huang C, Wang Y, Li X et al. Clinical features of patients infected with 2019 novel coronavirus in Wuhan, China. Lancet 2020;395:497-506

3. WHO. Coronavirus disease (COVID-19) outbreak. 2020. https:// www.who.int/emergencies/diseases/novel-coronavirus-2019. Accessed 20 Mar 2020

4. Bussani R, Schneider E, Zentilin L et al. Persistence of viral RNA, pneumocyte syncytia and thrombosis are hallmarks of advanced COVID-19 pathology. EBioMedicine. 2020;61:103104.

5. Carsana L, Sonzogni A, Nasr A et al. Pulmonary post-mortem findings in a series of COVID-19 cases from northern Italy: a twocentre descriptive study. Lancet Infect Dis. 2020;20:1135-1140. 
6. Environment NIfPHat. Generic framework for coronavirus measures. 2020. https://www.rivm.nl/en/novel-coronavirus-covid-19/ work-and-leisure/generic-framework-for-coronavirus-measures. Accessed 12 July 2020.

7. de Haas M, Faber R. Hamersma M. How COVID-19 and the Dutch 'intelligent lockdown' change activities, work and travel behaviour: Evidence from longitudinal data in the Netherlands. Transp Res Interdiscip Perspect 2020;6:100150.

8. Torres J, Mehandru S, Colombel JF et al. Crohn's disease. Lancet. 2017;389:1741-1755.

9. Ungaro R, Mehandru S, Allen PB et al. Ulcerative colitis. Lancet. 2017:389:1756-1770.

10. Sheriff MZ, Mansoor E, Luther J et al. Opportunistic infections are more prevalent in Crohn's disease and ulcerative colitis: a large population-based study. Inflamm Bowel Dis. 2020;26:291-300.

11. An P, Ji M, Ren $\mathrm{H}$ et al. Prevention of COVID-19 in patients with inflammatory bowel disease in Wuhan. China. Lancet Gastroenterol Hepatol. 2020;5:525-527.

12. Monteleone G, Ardizzone S. Are patients with inflammatory bowel disease at increased risk for covid-19 infection? J Crohns Colitis. 2020;14:1334-1336.

13. Knowles SR, Graff LA, Wilding H et al. Quality of life in inflammatory bowel disease: A systematic review and meta-analysespart I. Inflamm Bowel Dis. 2018;24:742-751.

14. Mikocka-Walus A, Knowles SR, Keefer L et al. Controversies revisited: A systematic review of the comorbidity of depression and anxiety with inflammatory bowel diseases. Inflamm Bowel Dis. 2016;22:752-762.

15. Gracie DJ, Hamlin PJ, Ford AC. The influence of the brain-gut axis in inflammatory bowel disease and possible implications for treatment. Lancet Gastroenterol Hepatol. 2019;4:632-642.

16. Brooks SK, Webster RK, Smith LE et al. The psychological impact of quarantine and how to reduce it: rapid review of the evidence. Lancet. 2020;395:912-920.

17. Irvine EJ. Development and subsequent refinement of the inflammatory bowel disease questionnaire: A quality-of-life instrument for adult patients with inflammatory bowel disease. J Pediatr Gastroenterol Nutr. 1999;28:S23-27.

18. Guyatt G, Mitchell A, Irvine EJ et al. A new measure of health status for clinical trials in inflammatory bowel disease. Gastroenterology. 1989;96:804-810.

19. Bodger K, Ormerod C, Shackcloth D et al. Development and validation of a rapid, generic measure of disease control from the patient's perspective: the IBD-control questionnaire. Gut. 2014;63:1092-1102.

20. Minnock P, Kirwan J, Bresnihan B. Fatigue is a reliable, sensitive and unique outcome measure in rheumatoid arthritis. Rheumatology (Oxford). 2009;48:1533-1536.

21. Team RC. R: A Language and Environment for Statistical Computing. 2020. https://www.r-project.org/. Accessed 13 Oct 2020.
22. Bates D, Mächler M, Bolker B et al. Fitting linear mixed-effects models using lme4. J Stat Softw 2015;67:48.

23. Rizopoulos D. Generalized linear mixed models using adaptive gaussian quadrature. 2020. https://drizopoulos.github.io/GLMMa daptive/reference/GLMMadaptive.html. Accessed 13 Oct 2020

24. Mollie E, Brooks KK, van Benthem KJ, Magnusson A, Berg CW, Nielsen A, Skaug HJ, Mächler M, Bolker BM. glmmTMB balances speed and flexibility among packages for zero-inflated generalized linear mixed modeling. $R J$ 2017;2017:378-400.

25. Pittet VEH, Maillard MH, Simonson T et al. Differences in outcomes reported by patients with inflammatory bowel diseases vs their health care professionals. Clin Gastroenterol Hepatol. 2019;17:2050-2059.

26. Shafer LA, Walker JR, Chhibba $T$ et al. Independent validation of a self-report version of the IBD disability index (IBDDI) in a population-based cohort of IBD patients. Inflamm Bowel Dis. 2018;24:766-774.

27. Grunert PC, Reuken PA, Stallhofer J, et al. Inflammatory bowel disease in the COVID-19 pandemic-The patients' perspective. J Crohns Colitis. 2020;14:1702-1708.

28. D'Amico F, Rahier JF, Leone S et al. Views of patients with inflammatory bowel disease on the COVID-19 pandemic: A global survey. Lancet Gastroenterol Hepatol. 2020;5:631-632.

29. Jones JL, Nguyen GC, Benchimol EI et al. The impact of inflammatory bowel disease in Canada 2018: quality of life. J Can Assoc Gastroenterol. 2019;2:S42-S48.

30. Taxonera C, Alba C, Olivares D, et al. Innovation in IBD care during the COVID-19 pandemic: Results of a cross-sectional survey on patient-reported experience measures. Inflamm Bowel Dis. 2021;27:864-869.

31. George LA, Cross RK. Remote monitoring and telemedicine in IBD: Are we there yet? Curr Gastroenterol Rep. 2020;22:12.

32. Azzam NA, Aljebreen A, Almuhareb A et al. Disability and quality of life before and during the COVID-19 outbreak: A crosssectional study in inflammatory bowel disease patients. Saudi $J$ Gastroenterol. 2020;26:256-262.

33. Yu M, Ye Z, Chen Y et al. Questionnaire assessment helps the self-management of patients with inflammatory bowel disease during the outbreak of Coronavirus disease 2019. Aging (Albany NY). 2020;12:12468-12478.

34. Pulvirenti F, Cinetto F, Milito C et al. Health-related quality of life in common variable immunodeficiency italian patients switched to remote assistance during the COVID-19 pandemic. J Allergy Clin Immunol Pract. 2020;8:1894-1899 e1892.

Publisher's Note Springer Nature remains neutral with regard to jurisdictional claims in published maps and institutional affiliations. 